\title{
Albumin-Bilirubin Grade and Hepatocellular Carcinoma Treatment Algorithm
}

Prof. M. Kudo

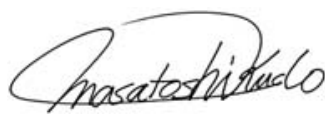

Editor Liver Cancer

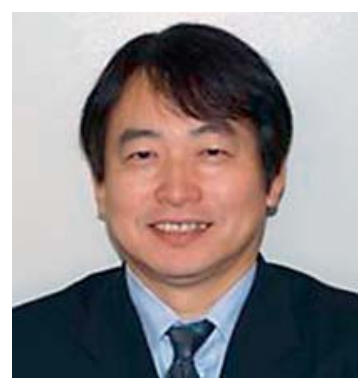

The selection of treatment options for hepatocellular carcinoma (HCC) requires critical consideration of hepatic functional reserve in addition to tumor burden (e.g., size and number of HCC nodules [tumor node metastasis stage]). Presently, the Child-Pugh classification is the most common worldwide measure used to assess hepatic functional reserve for the selection of treatment options for HCC [1]. The Child-Pugh classification is used in the American (AASLD [2]), European (EASL-EORTC [3]), Asian (APASL [4]), and Japanese (consensus-based [5, 6] and evidence-based [7-9]) guidelines.

However, there are several issues associated with the Child-Pugh scoring system. First, it includes the subjective criteria of hepatic encephalopathy and ascites in addition to albumin and bilirubin levels. Second, the classification of albumin levels and ascites together is not adequate because they are interrelated factors. Furthermore, converting prothrombin time into the international normalized ratio produces a score of 1 in many patients. Nonetheless, the Child-Pugh score remains the standard measure for selecting treatment options for HCC in clinical practice and clinical trials.

In Japan, a classification called the liver damage grade, which was developed by the Liver Cancer Study Group of Japan (LCSGJ), has been used historically instead of the Child-Pugh score [10]. This classification resembles the Child-Pugh classification but replaces 1 of the 5 factors (hepatic encephalopathy) by the results from the indocyanine green retention test at 
Kudo et al.: Albumin-Bilirubin Grade and Hepatocellular Carcinoma Treatment Algorithm

15 min (ICG R15). It is considered a more accurate assessment of hepatic functional reserve than the Child-Pugh classification. The difference between the 2 systems is particularly marked when comparing candidates for hepatectomy; namely, patients with Child-Pugh class A and liver damage grade A. Studies show that Child-Pugh class A is broader and encompasses a larger patient population than liver damage grade A [11,12]. Liver damage grade is also superior to the Child-Pugh classification for determining whether hepatectomy is indicated [12]. The ICG R15, a factor in the liver damage grading system, is also important for determining the resectable area from the perspective of hepatic functional reserve [13-15]. Makuuchi et al. [15] established the Makuuchi criteria for determining the method of hepatectomy (enucleation, partial hepatectomy, subsegmentectomy, segmentectomy, or resection of 2 or more segments) based on the ICG R15 and total bilirubin values. The Makuuchi criteria enable a safe hepatectomy; therefore, this approach has become the gold standard for selecting the method of hepatectomy not only in Japan $[15,16]$ but also in other countries [17]. However, there are problems associated with the liver damage grade. First, obtaining ICG R15 values requires injection of ICG in addition to blood collection. Second, injection of ICG for the ICG test can induce anaphylactic shock. Third, accurate values cannot be calculated for patients with marked constitutional jaundice or a portosystemic shunt. Therefore, the ICG R15 test is rarely performed prior to nonsurgical treatments, such as radiofrequency ablation or transcatheter arterial chemoembolization, in patients with relatively good liver function, even in Japan. This is also why the Japanese Evidence-Based Clinical Practice Guidelines list both the Child-Pugh classification and liver damage grade as methods for assessing hepatic functional reserve. The liver damage grade is useful for determining whether hepatectomy is indicated and for determining the size of the liver allowing resection, but it is not useful for the application of nonsurgical treatment.

The albumin-bilirubin (ALBI) grade was proposed as a means of overcoming the previously described issues with the Child-Pugh classification and liver damage grade by calculating a score based on recent albumin and bilirubin levels [18]. This has since then been the subject of many studies [19-25], with the conclusion that the ALBI grade is more accurate than the Child-Pugh classification for assessing hepatic functional reserve.

Hiraoka et al. [26] performed the first study comparing the ALBI grade with the LCSGJ liver damage grade, and the results were reported in this issue of Liver Cancer. Consistent with previous studies, Hiraoka et al. [26] showed that the ALBI grade was superior to the Child-Pugh classification. They also found that the ALBI grade can assess hepatic functional reserve with comparable efficacy to the liver damage grade. In addition, patients with ALBI scores of 1 and 2 showed a distribution of ICG R15 levels similar to that of patients with liver damage grades A and B. The ALBI score was well correlated with the ICG R15 $(r=0.616, p<$ 0.001), making it possible to roughly estimate the ICG R15 from the ALBI score. Based on the Makuuchi criteria, patient cohorts with an ICG R15 $<10,10-19$, and 20-30\% are considered candidates for two-segmentectomy, segmentectomy, and subsegmentectomy, respectively. Although the ICG R15 is an important factor for determining the resectable area, Hiraoka et al. [26] found that the ALBI score can also be used to identify groups corresponding to such ranges of ICG R15 values. Specifically, they found that the ALBI score for predicting an ICG R15 of $<10 \%$ was -2.623 (AUC, 0.798 ), the ALBI score for predicting an ICG R15 of 10-20\% was -2.470 (AUC, 0.791), and the ALBI score for predicting an ICG R15 of 20-30\% was -2.222 (AUC, 0.843). Although they noted that performing an ICG R15 test may be an additional option, particularly for patients who require resection, they showed that the ALBI score can fundamentally replace the liver damage grade, since the ICG R15 can be correctly predicted by the ALBI score.

In the Japanese evidence-based treatment algorithm [7], liver damage grade is used only when hepatectomy will be performed, whereas the Child-Pugh classification is used for 
patients receiving nonsurgical treatment [9, 27-29]. However, this is essentially a double standard, which is undesirable for clinical practice guidelines. Hiraoka et al. [26] proposed that the ALBI grade is a better measure to address this issue because the score is highly accurate for both nonsurgical and surgical treatments. This is a reasonable and clear-cut message.

In conclusion, the use of the ALBI grade instead of the Child-Pugh classification or liver damage grade to assess hepatic functional reserve for determining treatment options for HCC is a rational proposal not only in clinical practice but also as part of the guidelines. However, it may take some time for clinicians to widely adopt the ALBI grade in the clinical setting because it was only recently introduced into clinical practice.

\author{
Masatoshi Kudo, MD, PhD, Editor-in-Chief
}

\title{
References
}

1 Pugh RN, Murray-Lyon IM, Dawson JL, et al: Transection of the oesophagus for bleeding oesophageal varices. Br J Surg 1973;60:646-649.

2 Bruix J, Sherman M: Management of hepatocellular carcinoma: an update. Hepatology 2011;53:1020-1022.

3 LlovetJM, Ducreux M, et al: EASL-EORTC clinical practice guidelines: management of hepatocellular carcinoma. J Hepatol 2012;56:908-943.

4 Omata M, Lesmana LA, Tateishi R, et al: Asian Pacific Association for the Study of the Liver consensus recommendations on hepatocellular carcinoma. Hepatol Int 2010;4:439-474.

5 Kudo M, Matsui O, Izumi N, et al: JSH consensus-based clinical practice guidelines for the management of hepatocellular carcinoma: 2014 update by the Liver Cancer Study Group of Japan. Liver Cancer 2014;3:458-468.

6 Kudo M, Matsui O, Izumi N, et al: Transarterial chemoembolization failure/refractoriness: JSH-LCSGJ criteria 2014 update. Oncology 2014;87(suppl 1):22-31.

7 Kokudo N, Hasegawa K, Akahane M, et al: Evidence-based clinical practice guidelines for hepatocellular carcinoma: the Japan Society of Hepatology 2013 update (3rd JSH-HCC guidelines). Hepatol Res 2015;45.

8 The Liver Cancer Study Group of Japan: The criteria for the evaluation of direct treatment effects in hepatocellular carcinoma (in Japanese). Acta Hepatol Jpn 2004;45:380-385.

9 Kudo M: Clinical practice guidelines for hepatocellular carcinoma differ between Japan, United States, and Europe. Liver Cancer 2015; 4:85-95.

10 Liver Cancer Study Group of Japan: General Rules for the Clinical and Pathological Study of Primary Liver Cancer, 3rd English edition. Tokyo, Kanehara \& Co, Ltd, 2010.

11 Chung H, Kudo M, Haji S, et al: A proposal of the modified liver damage classification for hepatocellular carcinoma. Hepatol Res 2006;34:124-129.

12 Omagari K, Ohba K, Kadokawa Y, et al: Comparison of the grade evaluated by "liver damage" of Liver Cancer Study Group of Japan and Child-Pugh classification in patients with hepatocellular carcinoma. Hepatol Res 2006;34:266-272.

13 Greco E, Nanji S, Bromberg IL, et al: Predictors of peri-opertative morbidity and liver dysfunction after hepatic resection in patients with chronic liver disease. HPB (Oxford) 2011;13:559-565.

14 Lau H, Man K, Fan ST, et al: Evaluation of preoperative hepatic function in patients with hepatocellular carcinoma undergoing hepatectomy. Br J Surg 1997;84:1255-1259.

15 Makuuchi M, Kosuge T, Takayama T, et al: Surgery for small liver cancers. Semin Surg Oncol 1993;9:298-304.

16 Imamura H, et al: One thousand fifty-six hepatectomies without mortality in 8 years. Arch Surg 2003;138: 1198-1206; discussion 1206.

17 Torzilli G, Makuuchi M, Inoue K, et al: No-mortality liver resection for hepatocellular carcinoma in cirrhotic and noncirrhotic patients: is there a way? A prospective analysis of our approach. Arch Surg 1999;134:984992.

18 Johnson PJ, Berhane S, Kagebayashi C, et al: Assessment of liver function in patients with hepatocellular carcinoma: a new evidence-based approach - the ALBI grade. J Clin Oncol 2015;33:550-558.

19 Wang YY, Zhong JH, Su ZY, et al: Albumin-bilirubin versus Child-Pugh score as a predictor of outcome after liver resection for hepatocellular carcinoma. Br J Surg 2016, Epub ahead of print.

20 Toyoda H, Lai PB, O’Beirne J, et al: Long-term impact of liver function on curative therapy for hepatocellular carcinoma: application of the ALBI grade. Br J Cancer 2016;114:744-750.

21 Hiraoka A, Kumada T, Nouso K, et al: Proposed new sub-grouping for intermediate-stage hepatocellular carcinoma using albumin-bilirubin grade. Oncology 2016;91:153-161.

22 Hiraoka A, Kumada T, Michitaka K, et al: Usefulness of albumin-bilirubin grade for evaluation of prognosis of 2,584 Japanese patients with hepatocellular carcinoma. J Gastroenterol Hepatol 2016;31:1031-1036. 
23 Edeline J, Blanc JF, Johnson P, et al: A multicentre comparison between Child Pugh and albumin-bilirubin scores in patients treated with sorafenib for hepatocellular carcinoma. Liver Int 2016;36:1821-1828.

24 Chan AW, Kumada T, Toyoda H, et al: Integration of albumin-bilirubin (ALBI) score into Barcelona Clinic Liver Cancer (BCLC) system for hepatocellular carcinoma. J Gastroenterol Hepatol 2016;31:1300-1306.

25 Chan AW, Chong CC, Mo FK, et al: Incorporating albumin-bilirubin grade into the cancer of the liver Italian program system for hepatocellular carcinoma. J Gastroenterol Hepatol 2017;32:221-228.

26 Hiraoka A, Kumada T, Kudo M, et al: Albumin-bilirubin (ALBI) grade as part of the Japan Society of Hepatology: a comparison with liver damage and Child-Pugh classifications. Liver Cancer 2017;6:204-215.

27 Kudo M: Surveillance, diagnosis, treatment, and outcome of liver cancer in Japan. Liver Cancer 2015;4:39-50.

28 Kudo M: Locoregional therapy for hepatocellular carcinoma. Liver Cancer 2015;4:163-164.

29 Tsurusaki M, Murakami T: Surgical and locoregional therapy of HCC: TACE. Liver Cancer 2015;4:165-175. 